\title{
Nonrandom sister chromatid segregation of sex chromosomes in Drosophila male germline stem cells
}

\author{
Yukiko M. Yamashita
}

(C) Springer Science+Business Media Dordrecht 2013

\begin{abstract}
Sister chromatids are the product of DNA replication, which is assumed to be a very precise process. Therefore, sister chromatids should be exact copies of each other. However, reports have indicated that sister chromatids are segregated nonrandomly during cell division, suggesting that sister chromatids are not the same, although their DNA sequences are the same. Researchers have speculated that stem cells may retain template strands to avoid replicationinduced mutations. An alternative proposal is that cells may segregate distinct epigenetic information carried on sister chromatids. Recently, we found that Drosophila male germline stem cells segregate sister chromatids of $\mathrm{X}$ and $\mathrm{Y}$ chromosomes with a strong bias. We discuss this finding in relation to existing models for nonrandom sister chromatid segregation.
\end{abstract}

Keywords stem cells sex chromosome . asymmetric division

$\begin{array}{ll}\text { Abbreviation } & \\ \text { GSCs } & \text { germline stem cells } \\ \text { GB } & \text { gonialblast }\end{array}$

Responsible Editor: Helder Maiato and Yves Barral.

Y. M. Yamashita $(\bowtie)$

Life Sciences Institute, Center for Stem Cell Biology, Department of Cell and Developmental Biology, School of Medicine, Cellular and Molecular Biology Program, University of Michigan,

Ann Arbor, MI 48109, USA

e-mail: yukikomy@umich.edu

\begin{tabular}{|c|c|}
\hline CySCs & cyst stem cells \\
\hline JAK-STAT & $\begin{array}{l}\text { Janus kinase-signal transducer and } \\
\text { activator of transcription }\end{array}$ \\
\hline BrdU & 5-bromo-2'-deoxyuridine \\
\hline SUN-KASH & SUN (Sad1 and UNC-84) and \\
\hline & KASH (Klarsicht, ANC-1 and \\
\hline CO-FISH & $\begin{array}{l}\text { Syne/Nesprin homology) } \\
\text { chromosome-oriented fluorescent } \\
\text { in situ hybridization }\end{array}$ \\
\hline
\end{tabular}

\section{Introduction}

Although sister chromatids are precise copies of each other, nonrandom sister chromatid segregation has been reported in various cell types. Nonrandom sister chromatid segregation is a phenomenon in which two sister chromatids are distinctively segregated into two daughter cells. However, often due to technical limitations, the methods that distinguish two sister chromatids have not had sufficient resolution to distinguish various hypotheses surrounding nonrandom sister chromatid segregation. Difficulties in distinguishing two sister chromatids are inherent: Sister chromatids are meant to be exact copies of each other, and distinguishing them can be challenging. Essentially, all methods have relied on incorporation of labeled nucleotides or nucleotide analogs (such as BrdU) into DNA during replication, with subsequent detection of these labeled products to determine which cells inherited DNA with labeled nucleotides. Depending on the experimental scheme, labelcontaining sister chromatids may be older strands or newer strands: The labeled nucleotides are often 
administered after the establishment of stem cells, marking newer strands. In other cases, labeled nucleotides are incorporated into older strands by administering them during early development or repair period after injury, when stem cells are supposedly dividing symmetrically.

Another factor that complicates the experimental design is that DNA replication is semiconservative. Each chromatid contains two DNA helices (Watson and Crick), with one strand older than the other: The older strand served as a template in the last $\mathrm{S}$ phase. Therefore, examining nonrandom sister chromatid segregation means that one has to determine "which cell of the two sister cells inherited the sister chromatid that contained the oldest DNA strand as a template." This poses considerable technical challenge: It is necessary to identify sister cells that resulted from a cell division in which nucleotide analogs have been incorporated exactly two cell cycles earlier.

In addition to these inherent difficulties, identifying the correct cell types (for example, a rare stem cell population, among which only a rare population is supposed to be undergoing asymmetric division) and correct cell pairs (sister cell pairs that resulted from a single cell division) for analysis have always been a challenge. Moreover, to conclusively demonstrate that the nonrandom sister chromatid segregation is related to cell fate, the cell fates and directionality of sister chromatid segregation must correlate-for example, do template strands of DNA always go to the stem cell side? These technical limitations have often led to alternative interpretations to many experimental data, and nonrandom sister chromatid segregation has remained a controversial area of research.

Hypotheses that explain nonrandom sister chromatid segregation

\section{Immortal strand hypothesis}

Immortal strand hypothesis proposes that stem cells may retain the template copy of DNA (more precisely, the sister chromatid that contains the oldest strand as a template) to avoid accumulation of DNA replicationinduced mutations (Cairns 1975; Fig. 1a). This idea has been extensively investigated in past years, resulting in many positive and negative results (Merok et al. 2002; Potten et al. 2002; Karpowicz et al. 2005; Smith 2005; Shinin et al. 2006; Conboy et al. 2007; Fei and Huttner
2009; Quyn et al. 2010; Schepers et al. 2011). Interestingly, there are also conflicting reports where two reports using the same cell type reached opposing conclusions. This is not necessarily a black-or-white issue, where one report is false, and the other is true. If only a rare population of cells is undergoing nonrandom sister chromatid segregation, slight differences in identifying certain cell types (although two reports were supposedly detecting the same cell type) might bias detection of the phenomenon.

\section{Transmitting distinct epigenetic information}

In parallel, it has been proposed that cells may segregate sister chromatid nonrandomly to transmit distinct epigenetic information (Fig. 1b). This is tempting because it is obvious that epigenetic regulation is the essence of fate diversification, yet it remains a mystery how cells can customize an epigenetic landscape for each cell. Studies on epigenetic regulation have mainly focused on the importance of "epigenetic enzymes" such as DNA methyltransferases and histone-modifying enzymes and their loss-of-function phenotypes, demonstrating the importance of these molecules and thus the importance of epigenetic regulation. In addition, genome-wide studies have uncovered cell-type specific patterns of DNA methylation, histone modifications, and chromatin regulator association, correlating the cell fates and epigenetic changes. However, the fundamental "chicken-or-egg" mystery remains to be answered: Exactly when is the epigenetic information acquired for a cell to adopt a specific fate? For example, does a cell know that it is going to adopt a skin cell fate thus prepare such epigenetic information (at least seeding epigenetic information) before it actually becomes a skin cell? If epigenetic information is prepared in cells before they adopt their actual fates, what is the mechanism to prepare epigenetic information for the future fate of the cell? Alternatively, does a cell adopt skin cell epigenetic information because it is already a skin cell? If so, it leads to a further question as to whether a cell can be a skin cell before having such epigenetic information. If so, what establishes their identity in the first place, if not epigenetic?

Nonrandom sister chromatid segregation can provide the means for cells to transmit distinct epigenetic information during asymmetric cell division before their fates are irreversibly determined. In this case, 
Fig. 1 Hypotheses that explain nonrandom sister chromatid segregation. a The immortal strand hypothesis proposes that the template copy of the sister chromatids with fewer replication-induced mutations may be retained in the stem cells. b An alternative hypothesis to explain nonrandom sister chromatid segregation. Distinct epigenetic information is transmitted to daughter cells through nonrandom sister chromatid segregation
A

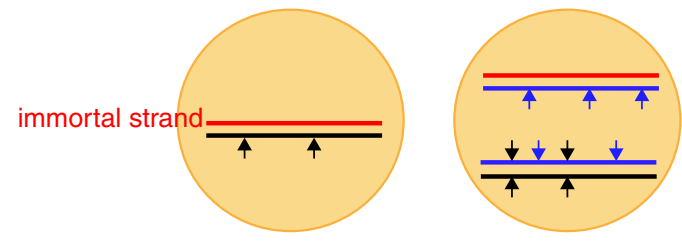

: Replication-induced mutation

\section{B}

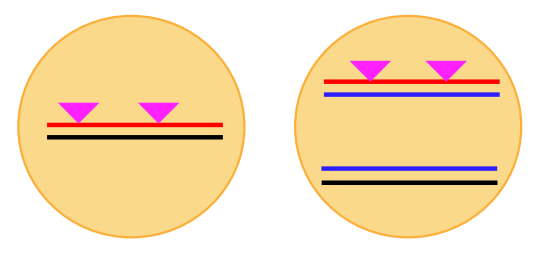

Asymmetric division
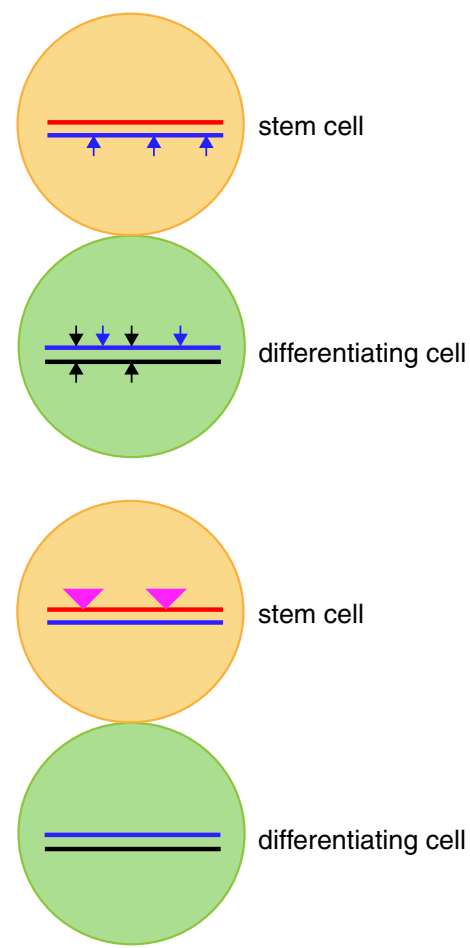

sister chromatids of all the chromosomes do not necessarily have to be distinguished. Instead, it is possible that only certain chromosomes with key fatedetermining genes might have to contain distinct epigenetic information and segregate asymmetrically. For example, when intestinal cells are dividing asymmetrically, a particular set of chromosomes (hypothetically, chromosomes 2, 11, and 18) might be segregated nonrandomly, whereas asymmetrically dividing neural stem cells might segregate a distinct set of chromosomes asymmetrically (hypothetically, chromosomes 5, 9, 14, and 22).

Drosophila male GSCs as a model system of asymmetric stem cell division

Drosophila male and female germline stem cells (GSCs) have served as premier model systems to study asymmetric stem cell division (Losick et al. 2011; Lehmann 2012). Male GSCs reside in the stem cell niche organized by hub cells. Hub cells secrete a ligand, unpaired (Upd), to activate the JAK-STAT pathway in the neighboring GSCs as well as in cyst stem cells (CySCs), a somatic stem cell population that shares the same niche with GSCs. Activation of JAK-STAT pathway in GSCs and CySCs specifies their stem cell identity (Kiger et al. 2001; Tulina and Matunis 2001; Leatherman and Dinardo 2008, 2010).

Under normal conditions, attachment to hub cells is the prerequisite for maintenance of GSC identity. Therefore, the asymmetric outcome of GSC division is governed by precisely regulated spindle orientation (Fig. 2a): Mitotic spindles of GSCs perpendicularly oriented toward the hub cells allow one daughter of GSC division to stay adhered to hub cells, leading to stem cell selfrenewal, and the other daughter to be displaced away from hub cells, leading to initiation of a differentiation program.

GSC spindle orientation is prepared by stereotypical positioning of the mother and daughter centrosomes during interphase. The mother centrosome always stays close to the hub cells, whereas the daughter migrates away from the hub cells, thereby preparing the perpendicularly oriented mitotic spindle (Yamashita et al. 2003, 2007). Although this simple cytoskeletal mechanism explains how GSCs prepare the mitotic spindle orientation, it has another implication: GSCs retain the oldest centrosome for a long time. This fact provoked the "immortal centrosome" hypothesis (Morrison and 


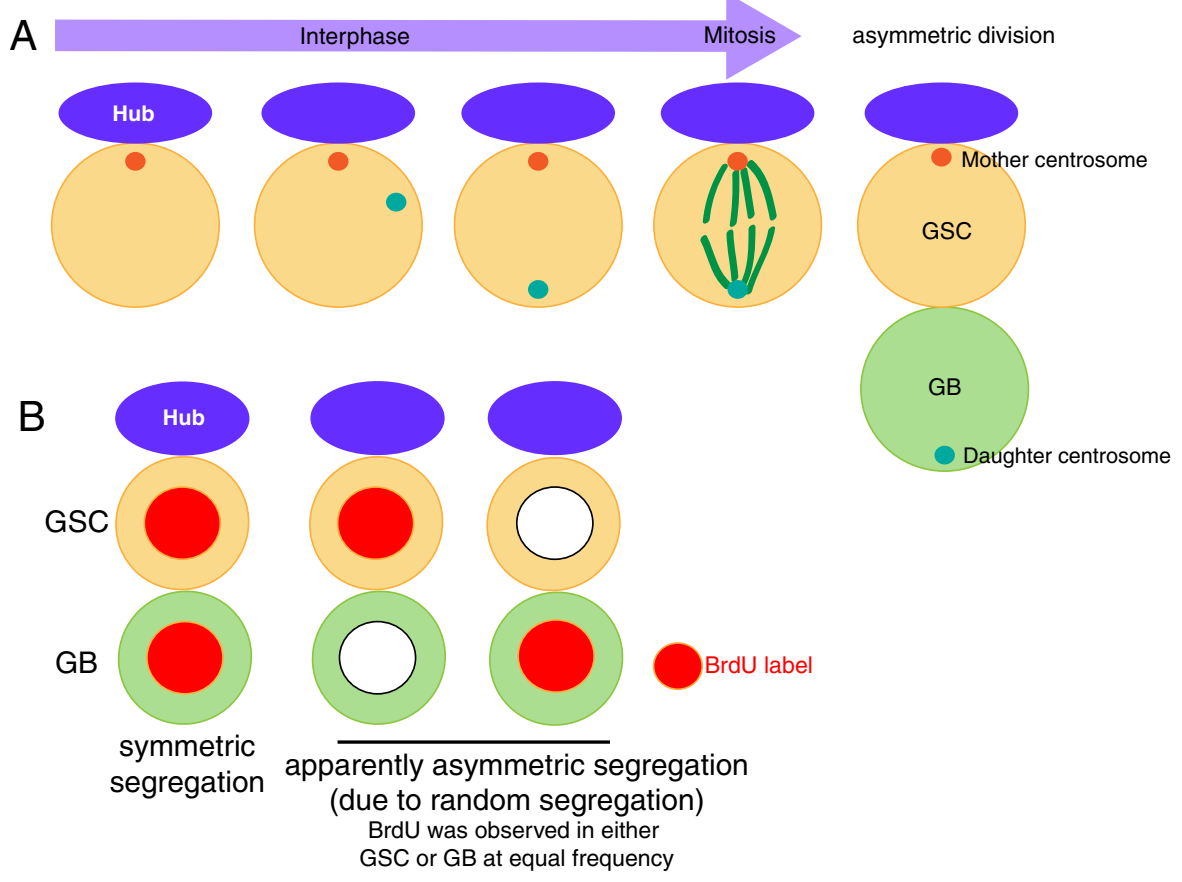

Fig. 2 Asymmetric stem cell division in Drosophila male germline stem cells (GSCs). a DROSOPHILA male GSCs divide asymmetrically by orienting the mitotic spindle with respect to the hub cells, which organize the stem cell niche. Spindle orientation is prepared by stereotypical movement of the mother and daughter centrosomes. As a result, the mother centrosome is retained in the GSCs for a prolonged time, whereas the daughter centrosome segregates into a differentiating daughter,

Spradling 2008) or "centrosomal eve" hypothesis (Yamashita and Fuller 2008), which speculated that the mother centrosome may harbor special information that confer or contribute to stem cell identity. One idea was that the mother centrosome might anchor the immortal DNA strand (Tajbakhsh and Gonzalez 2009).

Drosophila male GSCs do not follow the immortal strand model

Intrigued by speculation that the mother centrosome may anchor the immortal DNA strands, we tested the immortal strand hypothesis in Drosophila male GSCs (Yadlapalli et al. 2011). Male GSCs are ideally suited to test the immortal strand hypothesis for the following reasons:

1. GSCs can be identified with no ambiguity as opposed to many other systems in which the immortal strand hypothesis was tested. the gonialblast (GB). b Although male GSCs divide asymmetrically with the mother centrosome inherited by the stem cells, they do not segregate the immortal strands. BrdU labeling was segregated mostly symmetrically between GSC and GB when flies were fed with BrdU and the labeling was chased. In rare cases, BrdU labeling was observed to be exclusively inherited by one cell, but the directionality of BrdU segregation (to GSC or GB) was random, suggesting that this asymmetry is due to random segregation

2. GSC division is almost always asymmetric (Yamashita et al. 2003; Sheng and Matunis 2011), as opposed to many other systems in which stem cells are supposed to undergo symmetric and asymmetric divisions.

3. Stem cell-differentiating cell pairs can be easily identified in the GSC system, which is critical to accurately assess sister chromatid segregation pattern.

4. GSC cell cycle characteristics are well known, which provides precision to target the second cell cycle after nucleotide analog incorporation for the assessment of sister chromatid segregation pattern.

By conducting a BrdU pulse-chase experiment, we followed the segregation of old vs. new strands during multiple rounds of GSC divisions. Starting from the state where all GSCs have labeled DNA, the majority of cell divisions yielded symmetric 
BrdU distribution until the BrdU is finally completely diluted, and GSCs become BrdU negative. This finding strongly argues against the immortal strand hypothesis in male GSCs (Fig. 2b). However, because Drosophila contains only six major chromosomes (X, Y, 2 chromosome IIs, and 2 chromosome IIIs; chromosome IVs are too small to account for major BrdU distribution), the probability that one cell inherits all the BrdU chromosomes is not negligible. Indeed, we observed GSC-GB pairs in which BrdU was observed exclusively in one cell at the expected frequency. Importantly, however, it was random as to which cell (GSC or GB) contained all the BrdU, indicating that this process is indeed the result of random segregation, instead of immortal strand segregation.
Nonrandom sex chromosome segregation in Drosophila male GSCs

From the results described above, it is unlikely that male GSCs protect their genome by immortal DNA segregation. However, it remains possible that GSCs segregate only a subset of chromosomes nonrandomly, possibly to transmit epigenetic information. Recently, we have developed a chromosome-oriented fluorescence in situ hybridization (CO-FISH) protocol at a single chromosome resolution (Fig. 3a; Yadlapalli and Yamashita 2013). The CO-FISH method has been used to show that mammalian intestinal cells show a striking bias in segregation (Falconer et al. 2010). A similar bias was detected in mouse satellite cells using the same method (Rocheteau et al. 2012). In these cases, however, subtelomeric sequences were used as a probe, and thus,
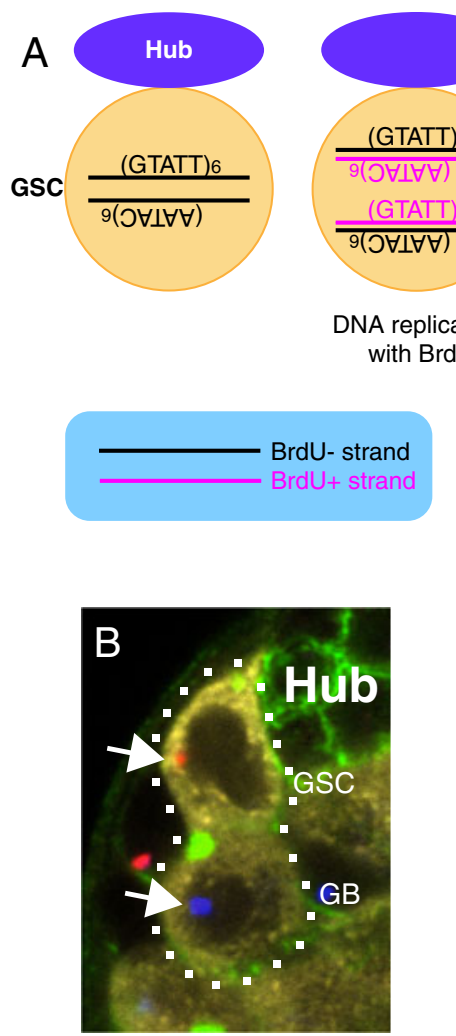

Fig. 3 Nonrandom sister chromatid segregation of $\mathrm{X}$ and $\mathrm{Y}$ chromosomes in Drosophila male GSCs. a The CO-FISH method to distinguish between sister chromatids. An example of a Drosophila Y chromosome containing (AATAC) $)_{6} /(\text { GTATT })_{6}$ is shown. Upon DNA replication in the presence of BrdU, only newly synthesized strands will contain BrdU. After fixation and irradiation with UV, BrdU-containing strands will be specifically nicked. Exonuclease III will remove the nicked strand, leaving the

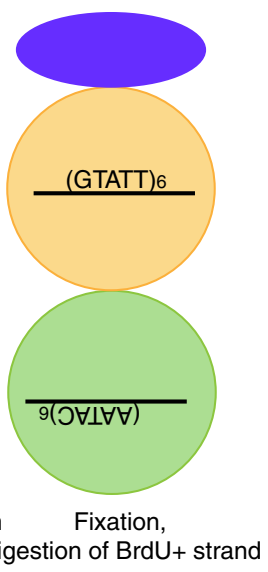

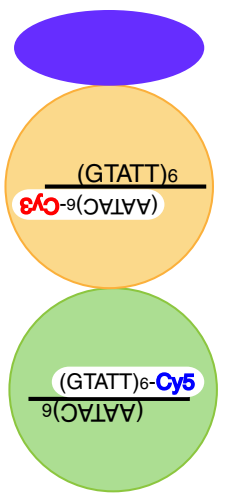

Hybridization with the probes

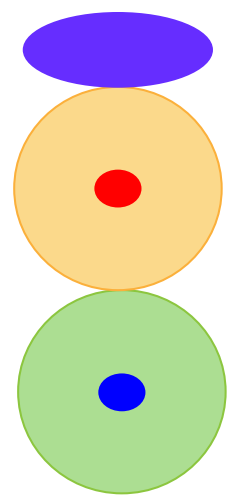

Detection of signals
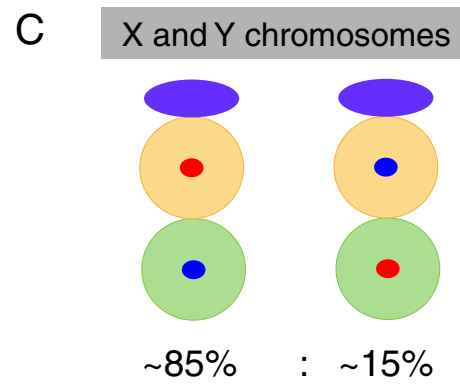

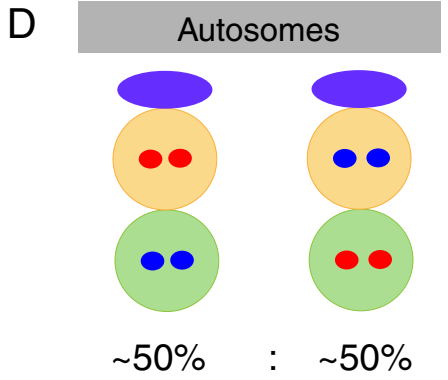

template strand intact. Upon hybridization of the CO-FISH probe, sister chromatids that contained (AATAC) 6 as a template vs. (GTATT) 6 as a template can be distinguished. b Example of CO-FISH signals observed in a GSC-GB pair using (AATAC) ${ }_{6}$-Cy3 (red) and (GTATT) ${ }_{6}-\mathrm{Cy} 5$ (blue; arrows). c Sister chromatid segregation pattern observed with X and Y chromosomes. d Sister chromatid segregation pattern observed with autosomes 
all chromosomes were detected simultaneously. Therefore, it is unclear whether certain chromosomes consistently show a bias, or the bias is always at the level of the population of chromosomes.

If a probe that is specific to a certain chromosome is used, two distinct strands can be detected at a single chromosome resolution. Combining CO-FISH method with chromosome-specific probes, we found that sex chromosomes are segregated nonrandomly during the asymmetric divisions of Drosophila male GSCs (Fig. 3b, c). For example, the Y chromosome contains $(\text { AATAC })_{6} /(\text { GTATT })_{6}$ repeats, which are unique to the $\mathrm{Y}$ chromosome but not to other chromosomes. By labeling (AATAC) 6 with Cy5, and $($ GTATT) 6 with $\mathrm{Cy} 3$, two sister chromatids of the Y chromosome can be distinguished (Fig. 3a). Using this method, we found that, in $85 \%$ of GSC divisions, GSC inherited the sister chromatid in which the template strand contains $(\text { GTATT })_{6}$ repeats (and thus hybridize to (AATAC) 6 $^{-}$ Cy3 probes). Additionally, in $15 \%$ of cases, GSCs inherited the sister chromatids in which the template strand contains (AATAC) ${ }_{6}$ repeats (Fig. 3b, c). For convenience, we will simply use "inheritance of $\mathrm{Cy} 3$ (red) or Cy5 (blue) signals" in the remainder of the text: "Inheritance of red signals" means that GSCs inherited the sister chromatid that has $(\text { GTATT })_{6}$ repeats as a template, which hybridized to the (AATAC) 6 -Cy3 probe, yielding a red signal after the CO-FISH procedure (see Fig. 3 for detail of CO-FISH methodology). A similar trend was observed $(\sim 85: 15)$ with $\mathrm{X}$ chromosome segregation. Despite a similar bias for $\mathrm{X}$ and $\mathrm{Y}$ chromosomes, we found that their segregation is independent of each other (Yadlapalli and Yamashita 2013).

Autosomes do not show biased sister chromatid segregation

Using the same CO-FISH procedure, we examined the segregation patterns of the autosomes. In contrast to $\mathrm{X}$ and $\mathrm{Y}$ chromosomes, which show a single CO-FISH signal/chromosome/cell, autosomes yield two CO-FISH signals/cell because cells contain two homologous chromosomes/cell-e.g., maternal chromosome II and paternal chromosome II. The first striking observation is that sister chromatids of two homologous chromosomes segregate together (Fig. 3d)-i.e., either two red signals or two blue signals were always observed in GSCs (Yadlapalli and Yamashita 2013). Because homologous chromosomes normally behave independently of each other in mitotic cells, this "co-segregation" is not necessarily expected and is an interesting phenomenon that would require explanation. It is possible that it is related to "homologous pairing" even in nonmeiotic cells observed in Drosophila and other dipterans (Fung et al. 1998). The relevance and elucidation of this "co-segregation" await future investigation.

Despite this unanticipated coordination between homologs, it seems to be random as to which signals (two reds or two blues) are inherited by GSCs: Approximately $50 \%$ of GSCs inherited two red signals, and the other approximately $50 \%$ of GSCs inherited two blue signals (Fig. 3d; Yadlapalli and Yamashita 2013). This trend was true with the second chromosome as well as the third chromosome. Although it is formally possible that approximately $50 \%$ of GSCs inherit a "red immortal strand" and the other remaining approximately $50 \%$ of GSCs retain a "blue immortal strand," we favor the possibility that autosome segregation is random as the simplest explanation. First, from our earlier study, male GSCs do not retain the immortal strand as a bulk (Yadlapalli et al. 2011). Second, even with $\mathrm{X}$ and $\mathrm{Y}$ chromosomes that show a striking bias in sister chromatid segregation at each division, we found that the strands are not immortal and each GSC switch the template strand every six to seven divisions, yielding 85:15 bias (Yadlapalli and Yamashita 2013). Again, it is still formally possible that any variation of immortal strand segregation may occur. For example, only the second chromosome is immortal, with half of the GSCs having a red immortal strand, and the other half retaining a blue immortal strand, whereas the third chromosome segregates completely randomly.

Mechanism of nonrandom sister chromatid segregation

In the previous studies that showed biased DNA strand segregation, such biased segregation was often correlated with the mode of stem cell division (such as symmetric vs. asymmetric, or selfrenewing vs. differentiating), providing insights into the underlying mechanisms that regulate a biased segregation pattern. However, it remains a mystery how biased sister chromatid segregation is achieved mechanistically: Even if stem cell identity or mode of division is controlling biased segregation, exactly how can cells distinguish 
between two sister chromatids and segregate them nonrandomly?

Our study showed that Cnn and SUN-KASH domain proteins are required for nonrandom sister chromatid segregation, providing the first insight into how cells might mechanistically achieve nonrandom sister chromatid segregation (Fig. 4a; Yadlapalli and Yamashita 2013). First, the requirement of the centrosome is in line with "the immortal centrosome hypothesis." It may seem that the requirement of the centrosome in this process is not surprising because the centrosome plays a fundamental role in chromosome segregation in general. However, it should be noted that centrosomes are not absolutely required for chromosome segregation to separate two complete sets of genomes into two daughters without chromosome missegregation. It is well known that chromosomes can undergo centrosomeindependent spindle formation to segregate chromosomes, with oocytes' acentrosomal meiosis in many species as the most prominent example. Strikingly, in Drosophila, a functional centrosome is not required at all for development of the animal (Megraw et al. 2001; Stevens et al. 2007). In the cnn mutant, cells can still segregate chromosomes equally into two daughter cells without causing obvious genomic instability. That said, the randomization of sister chromatid segregation observed in the cnn mutant

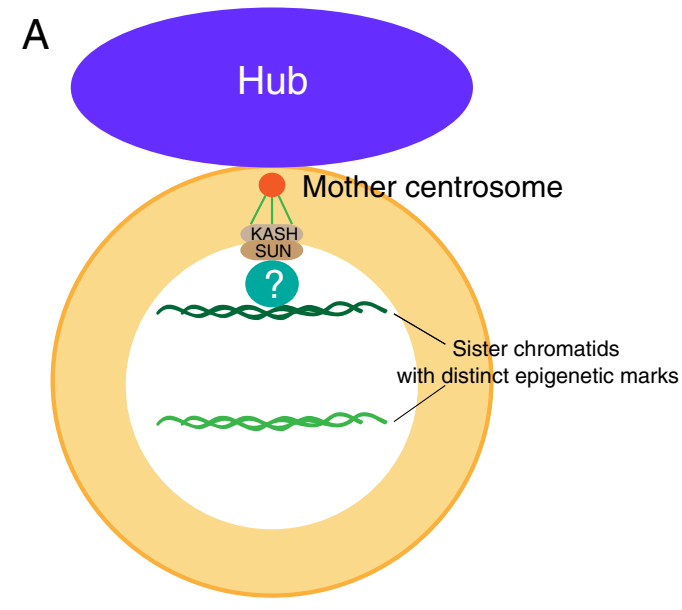

Fig. 4 Model for nonrandom sister chromatid segregation. a Molecular machineries that enable nonrandom sister chromatid segregation. Based on the requirement of cnn, koi, klar, and dnmt2, we propose that the certain sister chromatids (due to Dnmt2-dependent modification) of X and $\mathrm{Y}$ chromosomes are anchored to the SUN-KASH domain proteins, which in turn interact with the mother centrosome, leading to nonrandom is a specific problem in distinguishing two sister chromatids of $\mathrm{X}$ and $\mathrm{Y}$ chromosomes during stem cell division, instead of a problem in maintaining normal ploidy.

A tempting possibility that can be inferred from a specific defect in the cnn mutant is that two sister chromatids are distinct in their ability to organize/bind kinetochore microtubules (Maiato et al. 2004), and such sister kinetochores are captured by mother vs. daughter centrosomes that also differ in microtubuleorganizing activity (Fig. 4b). This idea is very similar to the "centromeric drive" hypothesis (Henikoff et al. 2001; Malik and Henikoff 2009), which proposes that distinct strength between paternal vs. maternal centromeres of the homologous chromosomes is used to gain access to oocyte (instead of being segregated into a polar body, which is an evolutionary dead end for a chromosome). It is speculated that maternal and paternal centromeres compete against each other by evolving their centromeric and pericentromeric sequences in a way to attract more kinetochore microtubules to gain access to the oocyte. However, too much imbalance between the paternal vs. maternal centromere leads to chromosome missegregation during mitosis; thus, the imbalance is counter-balanced by mutating centromeric/ pericentromeric-binding proteins that assist equal segregation of chromosomes. Although the basic idea of underlying cytoskeletal mechanisms is similar, there is

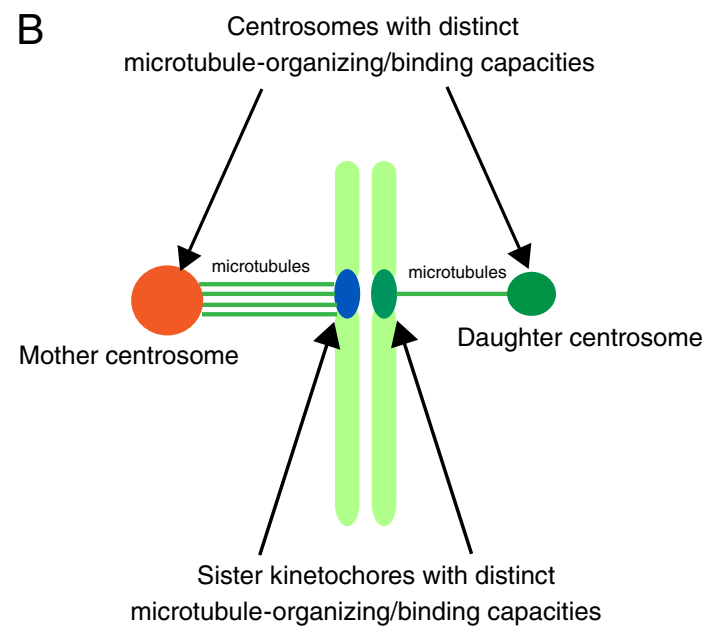

sister chromatid segregation. b A model to explain how two sister chromatids may be distinguished. Similar to the "centromeric drive" hypothesis, distinct kinetochore activities between two sister chromatids may be used to be captured by mother vs. daughter centrosomes, leading to nonrandom sister chromatid segregation 
a fundamental difference between the centromeric drive hypothesis and our model of nonrandom sister chromatid segregation: Because homologous chromosomes are not identical copies of each other, distinct centromeric strength can have a genetic basis, such as different copy numbers/sequences of centromere and/or pericentromeric regions. By contrast, explanation of nonrandom sister chromatid segregation cannot rely on different copy numbers or sequence of the centromere/pericentromeric regions because sister chromatids are identical copies of each other. Thus, if this idea of nonrandom sister chromatid segregation is correct, the difference should be purely epigenetic.

Although the idea that the centrosome captures specific sister chromatid is an attractive one, and indeed, it has been postulated as a basis for the immortal strand hypothesis, the centrosome does not directly interact with the chromosome in interphase because they are in distinct cellular compartments (cytoplasm and nucleus, respectively) separated by the nuclear envelope. SUNKASH domain proteins comprise the linker of nucleoskeleton and cytoskeleton (LINC) complex on the nuclear envelope and are known to mediate the interaction between the cytoplasm and nucleus (Razafsky and Hodzic 2009). KASH domain proteins are known to be on the outer nuclear membrane and interact with cytoskeleton components such as microtubules and actin filaments. Conversely, SUN domain proteins, which directly bind to KASH domain proteins, localize on the inner nuclear membrane and connect to chromatin. Such linkage between the cytoskeleton and chromosomes via the LINC complex is known to be required for multiple processes, such as meiotic homologous pairing and protecting the nucleus from the shearing force of cytoskeletons (Hiraoka and Dernburg 2009; Razafsky and Hodzic 2009). Thus, the requirement of SUN-KASH proteins in nonrandom sister chromatid segregation is not surprising, and it suggests that sister chromatids has to be distinguished throughout the cell cycle to achieve nonrandom sister chromatid segregation, instead of certain sister chromatids being successfully captured by the mother centrosome only during mitosis.

Dnmt2 is required for nonrandom sister chromatid segregation

In addition to the cytoskeletal components described above, we found that Dnmt2 is also required for nonrandom sister chromatid segregation (Yadlapalli and Yamashita 2013). dnmt2 is the sole gene in the Drosophila genome that potentially encodes DNA methyltransferase. Thus, it may seem natural to assume that Dnmt2 functions to provide distinct epigenetic marks between two sister chromatids, leading to nonrandom sister chromatid segregation. However, Dnmt2's functionality in Drosophila is still obscure, and thus, it remains unclear how Dnmt2 contributes to nonrandom sister chromatid segregation. Although some studies have suggested that Dnmt2 functions as a DNA methyltransferase (Kunert et al. 2003; Phalke et al. 2009), a more recent report has suggested that Dnmt2 may function as a transfer RNA methyltransferase (Schaefer et al. 2010). Moreover, a recent study using bisulfite sequencing showed that only $0.5 \%$ of the Drosophila genome is methylated, suggesting that DNA methylation might not be able to contribute to the distinction between sister chromatids (Zemach et al. 2010). However, these studies do not necessarily exclude the possibility that DNA methylation by Dnmt2 occurs only in a limited type of cell (instead of embryos that were used as the material for bisulfite sequencing) and/or in a manner that cannot be detected by bisulfate sequencing (e.g., methylation is only distributed to certain area, without strict sequence dependence). Future investigation is required to understand exactly how Dnmt2 contributes to nonrandom sister chromatid segregation.

Nevertheless, results of our recent study clearly suggests that Dnmt2 functions to provide "epigenetic information" to achieve nonrandom sister chromatid segregation, although the physical basis of "epigenetic information" may not be DNA methylation. Our study suggested that Dnmt2 indeed somehow primes the information during parents' gametogenesis, which can be inherited many cell generations (Yadlapalli and Yamashita 2013). For example, nonrandom sister chromatid segregation of $\mathrm{X}$ chromosomes in male GSCs depends on Dnmt2 function in the mother, whereas that of Y chromosome depends on Dnmt2 function in the father. Interestingly, in the same GSCs where Y chromosome is segregated randomly due to having a mutant father, the $\mathrm{X}$ chromosome that came from the wild-type mother was segregated with a normal bias ( 85:15). Likewise, the Y chromosome that came from the wild-type father was segregated normally with the bias when the segregation of the $\mathrm{X}$ chromosome from the mutant mother was 
randomized. These results reveal the striking possibility that the very first chromosome (the single copy of $\mathrm{X}$ from the mother and the single copy of $\mathrm{Y}$ from the father) possesses essential information that allows them to be inherited with the bias in the progeny's adult stem cells. The pattern of segregation is consistent with the idea that Dnmt2 prepares "epigenetic information" in germs cells in preparation for how chromosomes should behave in the progeny's GSCs.

Stem cell identity and nonrandom sister chromatid segregation

Is nonrandom sister chromatid segregation important for stem cell identity? Clearly, nonrandom sister chromatid segregation is not a determinant of stem cell identity because all mutants defective in nonrandom sister chromatid segregation do not lead to stem cell loss or stem cell tumors. Furthermore, these mutants are fertile, albeit at reduced fertility, except for cnn mutant that is sterile due to cytokinesis defects during meiosis (Yadlapalli and Yamashita 2013). This finding is not surprising considering that partially differentiated spermatogonia can revert to GSCs through the process of dedifferentiation (Brawley and Matunis 2004), although it is possible that dedifferentiated GSCs are less functional in producing sperms of equal quality and quantity compared to native GSCs. Whatever epigenetic information GSCs are transmitting to self-renewing daughters vs. differentiating daughters, it is likely that such information can be reverted on dedifferentiation, or at least the spermatogonial cells can override the instruction provided by the epigenetic information to re-acquire stem cell identity.

Nonetheless, we found that nonrandom sister chromatid segregation was compromised in tumor-like GSC overproliferation induced by overexpression of the "stemness factor" Upd, as well as upon dedifferentiation. Randomization in GSC overproliferation suggests that GSC identity is upstream of nonrandom sister chromatid segregation. Randomization upon dedifferentiation may not seem surprising; however, if one considers all other information carefully, it is somewhat puzzling and insightful at the same time. First, randomization upon dedifferentiation suggests that the sister chromatid that remains in the GSC contains special information, whereas the copy that was transmitted to the differentiating daughter does not have such information, explaining why dedifferentiated GSCs cannot regain nonrandom segregation - the information was lost forever. This finding is also in line with the data that the very first $\mathrm{X}$ and $\mathrm{Y}$ chromosomes that are transmitted from parents have seeding information. However, if this is the case, how can we interpret the data that $\mathrm{X}$ or $\mathrm{Y}$ chromosomes are not immortal and switch the copy every six to seven divisions on average? When the "template copies" (which had been a template in the last five to six divisions) switches at the sixth or seventh division, how can the epigenetic information be transmitted correctly? Another possibility is that, although dedifferentiated GSCs can re-establish the difference between two sister chromatids, the lack of a mother centrosome due to dedifferentiation (Cheng et al. 2008) might compromise nonrandom sister chromatid segregation. Clearly, there is a missing piece in this puzzle, and further investigation is required to address this problem.

Asymmetric histone segregation and nonrandom sister chromatid segregation

Recently, it was shown that male GSCs segregate old vs. newly synthesized histones nonrandomly (Tran et al. 2012). When the histone gene was converted from histone-GFP to histone-mKO, considerable asymmetry was observed in the subsequent GSC division, where GSCs inherited old histones (histone-GFP), whereas GB inherited new histones (histone-mKO). This is remarkable because it indicates that cells have an ability to coordinate the incorporation of histone molecules along the entire length of the chromosomes, and it could provide a mechanistic basis for transmission of epigenetic information in a well-regulated and well-coordinated manner. Because each chromosome likely has multiple replication origins and, thus, multiple replication forks, it is fascinating to imagine how cells might be able to coordinate which strand gets which histone (old or new). In this regard, the possibility that the replication machinery uses information on the DNA strand (e.g., the DNA strand is hemimethylated, serving as the basis to distinguish between the two strands) seems to be very appealing. However, the relationship between asymmetric histone segregation and nonrandom sister chromatid segregation is currently unknown. Are template DNA strands always associated with the old histones? Our study clearly shows that the template DNA strands do not 
necessarily remain in the GSCs. Does this finding indicate that histones and DNA strands behave completely independently? One consideration is that the experimental system employed by Tran et al. does not provide information regarding how often GSC division shows asymmetric histone segregation, although it is clear that old histones are segregated into GSCs whenever asymmetry is observed [i.e., Tran et al. (2012) never observed opposite asymmetry where all old histones are segregated into GBs]. To probe the relationship between asymmetric histone segregation and nonrandom sister chromatid segregation, it might be informative to examine histone segregation patterns in mutants that randomize sister chromatid segregation.

Why only X and Y chromosomes?

Why are only sex chromosomes segregated nonrandomly? At least in the model of GSCs, the "immortal strand hypothesis" to avoid the accumulation of DNA mutations is unlikely. This leaves us with the idea that distinct epigenetic information is transmitted to stem cells vs. differentiating daughters, although there may be other possibilities that have never been considered. Here, we are aware that this is somewhat a "chicken-or-egg" problem. From the requirement of Dnmt2 and the way it contributes to nonrandom sister chromatid segregation, we hypothesized that epigenetic information is the cause of nonrandom sister chromatid segregation. In speculating the "reason" for nonrandom sister chromatid segregation, we hypothesize that nonrandom sister chromatid segregation is the means of segregating distinct epigenetic information. It is possible that there are two types of epigenetic information: (1) certain epigenetic information that mechanistically allows nonrandom sister chromatid segregation-for example, those in the centromeric or pericentromeric area as described above-and (2) certain epigenetic information that are carried to distinct cell types via nonrandom sister chromatid segregation-for example, epigenetic marks on the genes that regulate cell fates.

In considering what information is carried to GSCs vs. GBs, using nonrandom sister chromatid segregation, the fact that only $\mathrm{X}$ and $\mathrm{Y}$ chromosomes show nonrandom sister chromatid segregation may provide a hint. Sex chromosomes are subjected to many epigenetic regulations, such as dosage compensation and male-specific sex chromosome inactivation, although whether these regulations operate in Drosophila male germline is still under the debate (Deng et al. 2011; Meiklejohn et al. 2011). The Drosophila Y chromosome is known to be highly heterochromatic and contains only a few known genes that generate transcripts (Piergentili and Mencarelli 2008). Those genes on Drosophila Y chromosomes are factors that are required for spermatogenesis, such as axonemal dynein. It is known that the precocious expression of these genes is toxic to nonspermatid cells, and its expression must be tightly suppressed except during spermiogenesis (likely including GSCs). In addition, the Drosophila X chromosome contains Stellate genes, which encode polypeptides, derepression of which is known to reduce fertility. Stellate repression normally occurs through the function of suppressor of Stellate $\mathrm{Su}(\mathrm{Ste})$, the gene encoding the Piwiinteracting RNA for Stellate, and $\mathrm{Su}(\mathrm{Ste})$ is located on the Y chromosome (Tulin et al. 1997; Aravin et al. 2001). These are examples of potential epigenetic regulation specific to $\mathrm{X}$ and $\mathrm{Y}$ chromosomes.

We found that Stellate is derepressed in mutants that are defective in nonrandom chromosome segregation. At this point, this result is only correlative, and how it correlates remains mystery. Defective nonrandom sister chromatid segregation is observed in GSCs, whereas derepression of Stellate occurs in spermatocytes and later stages. Thus, even if nonrandom sister chromatid segregation is indeed responsible for Stellate repression, it is still unclear how nonrandom sister chromatid segregation in GSCs contributes to Stellate repression in later stages of spermatogenesis. To obtain a definitive answer to this question, a mutant is required that is specifically defective in nonrandom sister chromatid segregation but not other processes. Currently, all mutants that are defective in nonrandom sister chromatid segregation (cnn, koi, klar, and dnmt2) have other defects as well, and it is impossible to conclude the direct relationship between nonrandom sister chromatid segregation and repression of Stellate.

\section{Conclusion and perspective}

The ability of Drosophila male GSCs to nonrandomly segregate sister chromatids of $\mathrm{X}$ and $\mathrm{Y}$ chromosomes demonstrate that it is possible that cells can distinguish 
between sister chromatids, which contain exactly the same genetic information. Identification of molecules involved in this process provides the framework for how nonrandom sister chromatid segregation is achieved mechanistically. Furthermore, those molecules are evolutionarily conserved, suggesting that they might serve the same function in other organisms. Future investigation will reveal how broadly this phenomenon is conserved and for what purpose it may serve.

\section{References}

Aravin AA, Naumova NM, Tulin AV, Vagin VV, Rozovsky YM, Gvozdev VA (2001) Double-stranded RNA-mediated silencing of genomic tandem repeats and transposable elements in the D. melanogaster germline. Curr Biol 11:1017-1027

Brawley C, Matunis E (2004) Regeneration of male germline stem cells by spermatogonial dedifferentiation in vivo. Science 304:1331-1334

Cairns J (1975) Mutation selection and the natural history of cancer. Nature 255:197-200

Cheng J, Turkel N, Hemati N, Fuller MT, Hunt AJ, Yamashita YM (2008) Centrosome misorientation reduces stem cell division during ageing. Nature 456:599-604

Conboy MJ, Karasov AO, Rando TA (2007) High incidence of non-random template strand segregation and asymmetric fate determination in dividing stem cells and their progeny. PLoS Biol 5:e102

Deng X, Hiatt JB, Nguyen DK, Ercan S, Sturgill D, Hillier LW, Schlesinger F, Davis CA, Reinke VJ, Gingeras TR, Shendure J, Waterston RH, Oliver B, Lieb JD, Disteche CM (2011) Evidence for compensatory upregulation of expressed X-linked genes in mammals, Caenorhabditis elegans and Drosophila melanogaster. Nat Genet 43:1179-1185

Falconer E, Chavez EA, Henderson A, Poon SS, McKinney S, Brown L, Huntsman DG, Lansdorp PM (2010) Identification of sister chromatids by DNA template strand sequences. Nature 463:93-97

Fei JF, Huttner WB (2009) Nonselective sister chromatid segregation in mouse embryonic neocortical precursor cells. Cereb Cortex 19(Suppl 1):i49-i54

Fung JC, Marshall WF, Dernburg A, Agard DA, Sedat JW (1998) Homologous chromosome pairing in Drosophila melanogaster proceeds through multiple independent initiations. J cell biol 141:5-20

Henikoff S, Ahmad K, Malik HS (2001) The centromere paradox: stable inheritance with rapidly evolving DNA. Science 293:1098-1102

Hiraoka Y, Dernburg AF (2009) The SUN rises on meiotic chromosome dynamics. Dev cell 17:598-605

Karpowicz P, Morshead C, Kam A, Jervis E, Ramuns J, Cheng V, van der Kooy D (2005) Support for the immortal strand hypothesis: neural stem cells partition DNA asymmetrically in vitro. J Cell Biol 170:721-732

Kiger AA, Jones DL, Schulz C, Rogers MB, Fuller MT (2001) Stem cell self-renewal specified by JAK-STAT activation in response to a support cell cue. Science 294:2542-2545

Kunert N, Marhold J, Stanke J, Stach D, Lyko F (2003) A Dnmt2-like protein mediates DNA methylation in Drosophila. Development 130:5083-5090

Leatherman JL, Dinardo S (2008) Zfh-1 controls somatic stem cell self-renewal in the Drosophila testis and nonautonomously influences germline stem cell selfrenewal. Cell Stem Cell 3:44-54

Leatherman JL, Dinardo S (2010) Germline self-renewal requires cyst stem cells and stat regulates niche adhesion in Drosophila testes. Nat Cell Biol 12:806-811

Lehmann R (2012) Germline stem cells: origin and destiny. Cell Stem Cell 10:729-739

Losick VP, Morris LX, Fox DT, Spradling A (2011) Drosophila stem cell niches: a decade of discovery suggests a unified view of stem cell regulation. Dev cell 21:159-171

Maiato H, Rieder CL, Khodjakov A (2004) Kinetochore-driven formation of kinetochore fibers contributes to spindle assembly during animal mitosis. J Cell Biol 167:831-840

Malik HS, Henikoff S (2009) Major evolutionary transitions in centromere complexity. Cell 138:1067-1082

Megraw TL, Kao LR, Kaufman TC (2001) Zygotic development without functional mitotic centrosomes. Curr Biol 11:116-120

Meiklejohn CD, Landeen EL, Cook JM, Kingan SB, Presgraves DC (2011) Sex chromosome-specific regulation in the Drosophila male germline but little evidence for chromosomal dosage compensation or meiotic inactivation. PLoS biology 9:e1001126

Merok JR, Lansita JA, Tunstead JR, Sherley JL (2002) Cosegregation of chromosomes containing immortal DNA strands in cells that cycle with asymmetric stem cell kinetics. Cancer Res 62:6791-6795

Morrison SJ, Spradling AC (2008) Stem cells and niches: mechanisms that promote stem cell maintenance throughout life. Cell 132:598-611

Phalke S, Nickel O, Walluscheck D, Hortig F, Onorati MC, Reuter G (2009) Retrotransposon silencing and telomere integrity in somatic cells of Drosophila depends on the cytosine-5 methyltransferase DNMT2. Nat Genet 41:696702

Piergentili R, Mencarelli C (2008) Drosophila melanogaster kl-3 and kl-5 Y-loops harbor triple-stranded nucleic acids. J Cell Sci 121:1605-1612

Potten CS, Owen G, Booth D (2002) Intestinal stem cells protect their genome by selective segregation of template DNA strands. J Cell Sci 115:2381-2388

Quyn AJ, Appleton PL, Carey FA, Steele RJ, Barker N, Clevers H, Ridgway RA, Sansom OJ, Nathke IS (2010) Spindle orientation bias in gut epithelial stem cell compartments is lost in precancerous tissue. Cell Stem Cell 6:175-181

Razafsky D, Hodzic D (2009) Bringing KASH under the SUN: the many faces of nucleo-cytoskeletal connections. J cell biol 186:461-472 
Rocheteau P, Gayraud-Morel B, Siegl-Cachedenier I, Blasco MA, Tajbakhsh S (2012) A subpopulation of adult skeletal muscle stem cells retains all template DNA strands after cell division. Cell 148:112-125

Schaefer M, Pollex T, Hanna K, Tuorto F, Meusburger M, Helm M, Lyko F (2010) RNA methylation by Dnmt2 protects transfer RNAs against stress-induced cleavage. Genes Dev 24:1590-1595

Schepers AG, Vries R, van den Born M, van de Wetering M, Clevers H (2011) Lgr5 intestinal stem cells have high telomerase activity and randomly segregate their chromosomes. Embo J.

Sheng XR, Matunis E (2011) Live imaging of the Drosophila spermatogonial stem cell niche reveals novel mechanisms regulating germline stem cell output. Development 138:3367-3376

Shinin V, Gayraud-Morel B, Gomes D, Tajbakhsh S (2006) Asymmetric division and cosegregation of template DNA strands in adult muscle satellite cells. Nat Cell Biol 8:677-687

Smith GH (2005) Label-retaining epithelial cells in mouse mammary gland divide asymmetrically and retain their template DNA strands. Development 132:681-687

Stevens NR, Raposo AA, Basto R, St Johnston D, Raff JW (2007) From stem cell to embryo without centrioles. Curr Biol 17:1498-1503

Tajbakhsh S, Gonzalez C (2009) Biased segregation of DNA and centrosomes - moving together or drifting apart? Nat Rev Mol Cell Biol 10:804-810
Tran V, Lim C, Xie J, Chen X (2012) Asymmetric division of Drosophila male germline stem cell shows asymmetric histone distribution. Science 338:679-682

Tulin AV, Kogan GL, Filipp D, Balakireva MD, Gvozdev VA (1997) Heterochromatic Stellate gene cluster in Drosophila melanogaster: structure and molecular evolution. Genetics 146:253-262

Tulina N, Matunis E (2001) Control of stem cell self-renewal in Drosophila spermatogenesis by JAK-STAT signaling. Science 294:2546-2549

Yadlapalli S, Yamashita YM (2013) Chromosome-specific nonrandom sister chromatid segregation during stem cell division. Nature (in press).

Yadlapalli S, Cheng J, Yamashita YM (2011) Drosophila male germline stem cells do not asymmetrically segregate chromosome strands. J Cell Sci 124:933-939

Yamashita YM, Fuller MT (2008) Asymmetric centrosome behavior and the mechanisms of stem cell division. J Cell Biol 180:261-266

Yamashita YM, Jones DL, Fuller MT (2003) Orientation of asymmetric stem cell division by the APC tumor suppressor and centrosome. Science 301:1547-1550

Yamashita YM, Mahowald AP, Perlin JR, Fuller MT (2007) Asymmetric inheritance of mother versus daughter centrosome in stem cell division. Science 315:518-521

Zemach A, McDaniel IE, Silva P, Zilberman D (2010) Genomewide evolutionary analysis of eukaryotic DNA methylation. Science 328:916-919 